\title{
Pregnancy Screening Strategies for Diagnostic Nuclear Medicine: Survey Results from Australia and New Zealand
}

\author{
Daphne J. James ${ }^{1}$, Paul Cardew ${ }^{1,2}$, and Helen M. Warren-Forward ${ }^{1}$ \\ ${ }^{1}$ School of Health Sciences, University of Newcastle, New South Wales, Australia; and ${ }^{2}$ Hunter New England Imaging, Hunter New \\ England Area Health Service, Newcastle, Australia
}

The ionizing radiation used in diagnostic nuclear medicine procedures has the potential to cause biologic harm to a fetus. Although the risks are relatively small, it is recommended that all female patients of childbearing age be questioned regarding their pregnancy status before administration of the radiopharmaceutical. This can be a sensitive situation especially for certain types of patients, such as teenagers. Currently, there are no guidelines that detail how to question the patient. Previous studies have revealed the lack of a consistent approach in this area. The aim of this study was to investigate current practice for pregnancy screening before diagnostic nuclear medicine procedures in Australia and New Zealand and to determine whether a standardized practice guideline is required. Methods: An online survey was administered via SurveyMonkey from October to December 2011. Members of the Australian and New Zealand Society of Nuclear Medicine were invited to participate. The survey consisted of 30 questions divided into 4 sections: demographics, policy and regulations, current practice, and open-ended clinical scenarios. Results: Three hundred thirty-five responses were recorded from participants in all states and territories of Australia and New Zealand; 90\% were nuclear medicine technologists. Participants reported a low awareness of radiation policy and regulations but demonstrated good knowledge of the relative risk to the fetus from commonly performed procedures. The most common minimum and maximum age to question patients was 12 y (32\%) and 55 y (42\%), respectively, although the range was from 10 to $60 \mathrm{y}$. Verbal questioning (44\%) was the most commonly used approach. Pregnancy testing was used by $72 \%$, usually if the patient indicated she was unsure of her pregnancy status. Responses to clinical scenarios were varied, and these will be discussed in a subsequent paper. Conclusion: The survey revealed a lack of awareness of government regulations and departmental policy regarding radiation protection. The study demonstrated wide variety in pregnancy screening strategies used to determine the pregnancy status of patients before diagnostic nuclear medicine procedures, indicating that a standardized practice guideline is required for Australia and New Zealand.

Key Words: ionizing radiation; pregnancy; fetal exposure

J Nucl Med Technol 2013; 41:216-222

DOI: 10.2967/jnmt.113.123216

Received Apr. 3, 2013; revision accepted Jun. 4, 2013.

For correspondence or reprints contact: Daphne James, School of Health Sciences, University of Newcastle, University Dr., Callaghan New South Wales 2308 Australia.

E-mail: Daphne.James@newcastle.edu.au

Published online Aug. 12, 2013.

COPYRIGHT (C) 2013 by the Society of Nuclear Medicine and Molecular Imaging, Inc.
$\mathbf{R}$ adioactive materials that emit ionizing radiation are used in nuclear medicine to perform diagnostic imaging procedures and therapeutic dose administration. Measures for the protection of the fetus are necessary because ionizing radiation has the potential to cause biologic damage in humans, and fetal tissue is particularly sensitive to these effects (1). The potential biologic effects from irradiating a fetus with ionizing radiation can be "teratogenic, mutagenic or carcinogenic" (2).

Therapeutic nuclear medicine entails the administration of large doses of a radiopharmaceutical designed to destroy specific cells, whereas diagnostic nuclear medicine procedures involve considerably smaller doses to image the physiology and anatomy of the body. It is imperative to determine whether a female patient could be pregnant before administration of the therapeutic dose to protect the fetus. Guidelines published by government and regulatory bodies, such as the International Commission on Radiological Protection (ICRP) (publication 84, Pregnancy and Medical Radiation (3)) and the Australian Radiation Protection and Nuclear Safety Agency (ARPANSA) (14.2, Safety Guide for Radiation Protection in Nuclear Medicine (4)), contain instructions on how to determine the pregnancy status of the patient before radiopharmaceutical dose administration. Because of the size of the doses used, pregnancy testing within $24 \mathrm{~h}$ of therapy administration is mandatory to ensure any unborn fetus is not irradiated (4). However, for diagnostic imaging procedures, where the amount of radiation administered is considerably smaller and the risk to the fetus is less, the guidelines are less clear. It is recommended that all women of childbearing age be questioned regarding their pregnancy status before diagnostic imaging procedures and that signs be placed around nuclear medicine departments asking patients to notify staff if they think they may be pregnant (4). However, there are no instructions on how to question the patient or how to determine which patients should be classed as within childbearing age.

In 2008, the American College of Radiology developed a practice guideline to provide information regarding fetal risk from ionizing radiation and instructions to assist radiographers on how to screen for pregnancy before radiologic examinations (5). These guidelines did not include information for nuclear medicine procedures. In 
2009, a review of European procedures suggested that there was a lack of consistent practice in this area and that more research was required (6).

In 2011, James et al. conducted an interview study in Australia to identify the methods used to question female patients about their pregnancy status before diagnostic nuclear medicine procedures and any problems that nuclear medicine scientists (NMSs) associated with these methods. The study reported that a variety of approaches to questioning were used in different departments, and even within departments, and that the most common form of questioning was a verbal approach, with the NMSs often using their discretion as to which patients to question (7). A limited number of interviews were conducted; therefore, the authors decided to investigate whether the findings were representative of current practice in nuclear medicine across Australia and New Zealand. A questionnaire was developed from the findings to investigate knowledge of pertinent regulations, methods of questioning, determination of age range for screening, use of pregnancy testing, and knowledge of fetal risk from ionizing radiation. The questionnaire was used to conduct a nationwide survey of nuclear medicine personnel in Australia and New Zealand.

The aim of the current study was to investigate current practice for pregnancy screening before diagnostic nuclear medicine procedures in Australia and New Zealand and to determine whether a standardized practice guideline is required.

\section{MATERIALS AND METHODS}

Ethics approval was gained from the University of Newcastle Human Research Ethics Committee before the study commenced (approval number H-2009-0270).

A SurveyMonkey questionnaire (supplemental data file; supplemental materials are available at http://jnm.snmjournals.org) consisting of 30 items divided into 4 sections was developed from the findings of the interview study (7). Questions in sections 1-3 were mainly closed responses, whereas section 4 sought openended responses to 4 clinical scenario questions. In section 1 , the demographic questions (items 1-8) asked about sex, profession, years of experience, and place of practice to help categorize participants and determine whether the study sample was representative of the nuclear medicine professional community in Australia and New Zealand. Section 2 (items 9-13) investigated participants' awareness of departmental policy and government regulations relating to pregnancy and medical radiation. Section 3 (items 14-25) investigated current practice in determining pregnancy status in nuclear medicine. Participants were asked to nominate the minimum and maximum ages that they routinely questioned their patients and give a rationale for this age. The questions asked which method of questioning was used-verbal, verbal with a signature, or a written form-and whether any questions about last menstrual period (LMP), contraceptive use, or menopause and hysterectomy were included. Participants were asked if they used pregnancy tests in their department and how often they were used, which type (serum or urine) was used, and in which circumstances they were used. Question 25 asked participants to rank 8 diagnostic procedures in order of risk to a fetus from the exposure to ionizing radiation from each procedure.

Section 4 (items 26-30) included 4 open-response questions on how to question the patient in a series of clinical scenarios and 1 open-response asking for any additional comments. The results from this section will be discussed in a subsequent paper.

The questionnaire was administered as an online survey via SurveyMonkey and was open for 2 mo from October 11 to December 12, 2011. An invitation to participate was published in the September 2011 issue of the Gamma Gazette (the journal of the Australian and New Zealand Society of Nuclear Medicine [ANZSNM]). It was also emailed to members of the ANZSNM with a link to the survey. In April 2011, there were 1,115 members of the ANZSNM, of which 839 were NMSs (J. Anderson, written e-mail communication, 2011).

Data analysis was performed using descriptive statistics, and open-ended responses were manually coded using thematic analysis.

\section{RESULTS}

\section{Demographics}

Three hundred thirty-five responses were recorded, and $66 \%$ were from women. Most participants were NMSs (90\%). The remainder consisted of 6 physicists and 33 nuclear medicine physicians. Most participants were experienced nuclear medicine professionals, with almost half (48\%) reporting that they had more than $10 \mathrm{y}$ of experience in their profession and $88 \%$ with more than $3 \mathrm{y}$ of experience. The type of practice that participants were in was relatively evenly split between public (48\%) and private $(52 \%)$ practice departments. Most participants practiced in New South Wales (38\%) and Victoria $(25 \%)$, although responses were recorded from all states and territories of Australia and New Zealand. The survey participants are believed to be representative of the nuclear medicine community in Australia and New Zealand. (J. Anderson, written e-mail communication, 2011).

\section{Policy and Regulations}

In response to the question asking if they were aware of a written policy regarding checking for pregnancy before diagnostic procedures in their department, 65\% (193/295) indicated yes, with $63 \%(121 / 193)$ having read the policy within the last 12 mo (Fig. 1). When asked if they were aware of any government regulations regarding how to determine the pregnancy status of patients before diagnostic imaging procedures, only 28\% (85/298) answered yes. Participants who answered that they were aware of government regulations were asked to briefly state, in their own words, what was included in the regulations (Fig. 2). Participants were then asked if they had read relevant sections in either ICRP 84 (3) or ARPANSA 14.2 (4). Most participants (76\% and $64 \%$, respectively) indicated that they had not read these documents.

\section{Current Practice}

Age. The most common minimum age reported to be used for questioning a patient about pregnancy was $12 \mathrm{y}$, 


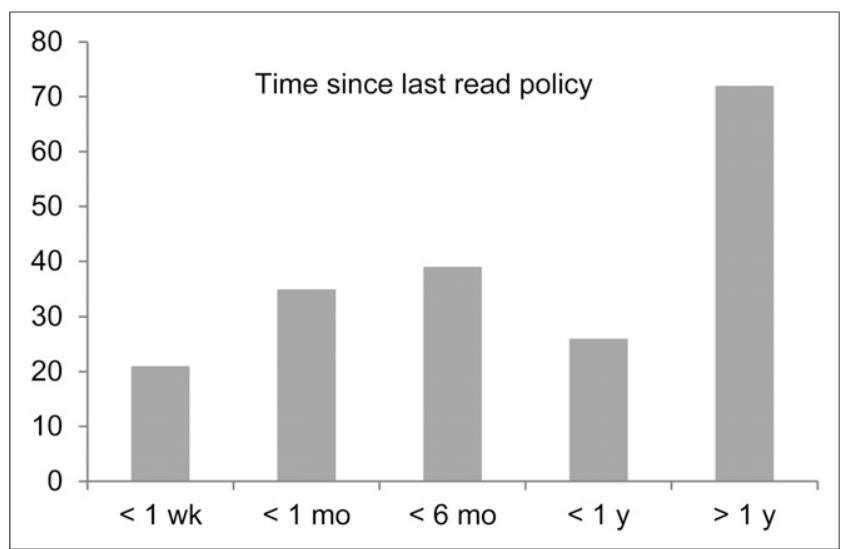

FIGURE 1. Time since participants had read department policy.

and the most common maximum age was $55 \mathrm{y}$. The age range for questioning was $10-60 \mathrm{y}$. The number of responses for each age category for male and female participants is displayed in Figure 3. There was no significant difference between the male and female responses $(P=0.08)$.

Participants were asked to state a rationale for using a particular minimum and maximum age to question their female patients about pregnancy. For the minimum age, 6 participants stated it was departmental policy, 6 had personal experience with a girl being pregnant at that age, and 18 said it depended on the maturity of the patient. The following were the most commonly used rationales for minimum age: age of menstruation (113), sexually active at this age (33), and at this age they would ask if the patient is menstruating first (21) (Fig. 4).

For the maximum age to question patients, 10 participants stated it was departmental policy and 7 had personal experience with a woman being pregnant at that age. The most commonly used rationales for maximum age were menopause (110) or unlikelihood of pregnancy at that age (26) (Fig. 5).

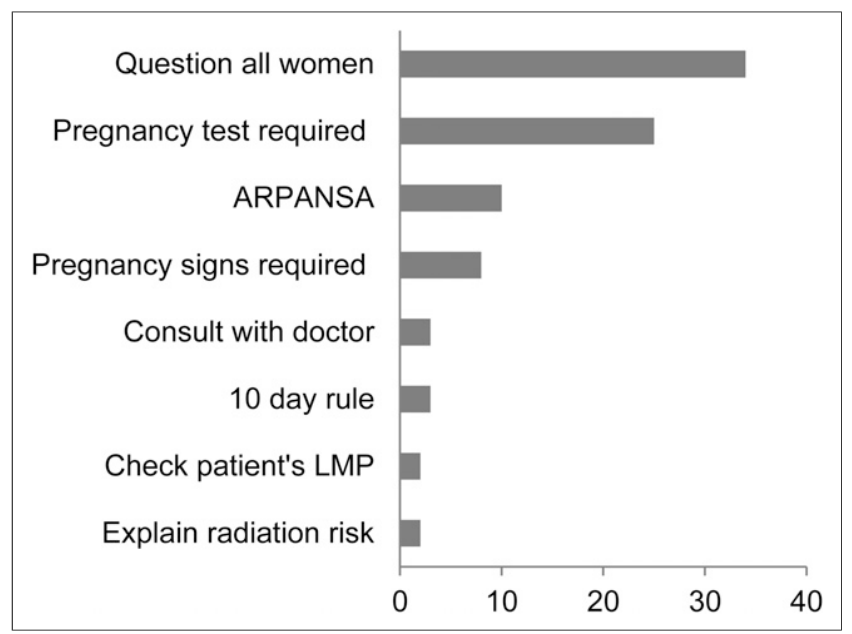

FIGURE 2. Open responses for content of regulations.

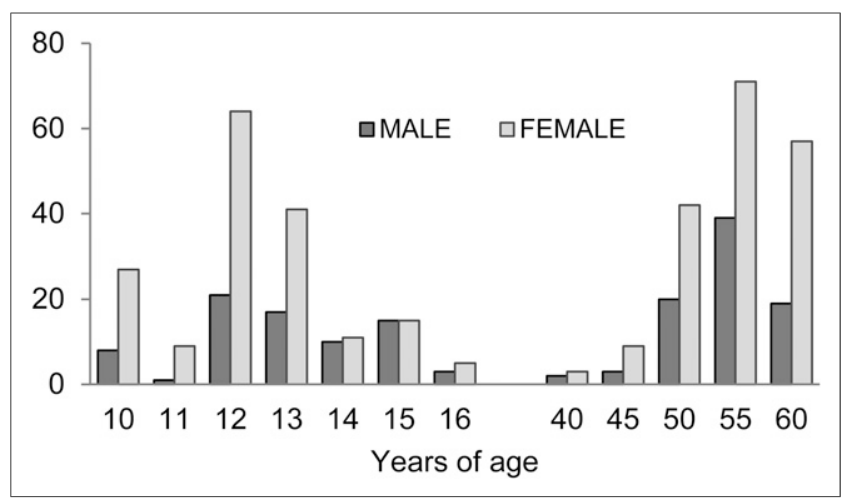

FIGURE 3. Age of questioning.

Method of Questioning. The most common method for determining pregnancy status was the use of verbal questioning (44\%), followed by verbal questioning with the addition of the patient's signature (35\%). Only 66 participants $(21 \%)$ used a written form to ask the patients about their pregnancy status. Assuming that the written forms require the patient's signature, most patients (56\%) are asked to verify that they have been questioned about their pregnancy status by providing their signature. Participants were asked to indicate if they used questions regarding LMP, contraceptive use, or menopause or hysterectomy history as part of their routine verbal or written questions. Questions about LMP were most commonly asked (89\% if verbal; $93 \%$ on written form). Participants using verbal questioning were more likely to ask questions about contraceptive use and menopause or hysterectomy than those who used a written form (Table 1).

Use of Pregnancy Tests. Of 262 responses, $72 \%$ indicated that they used pregnancy tests in their department. Serum testing (69\%) was most commonly used, with $50 \%$ using urine tests. The shared percentages are greater than $100 \%$ because some respondents ticked both serum and urine testing. Participants were asked to state in their own words in which circumstances they would use pregnancy tests.



FIGURE 4. Rationale for minimum age. 


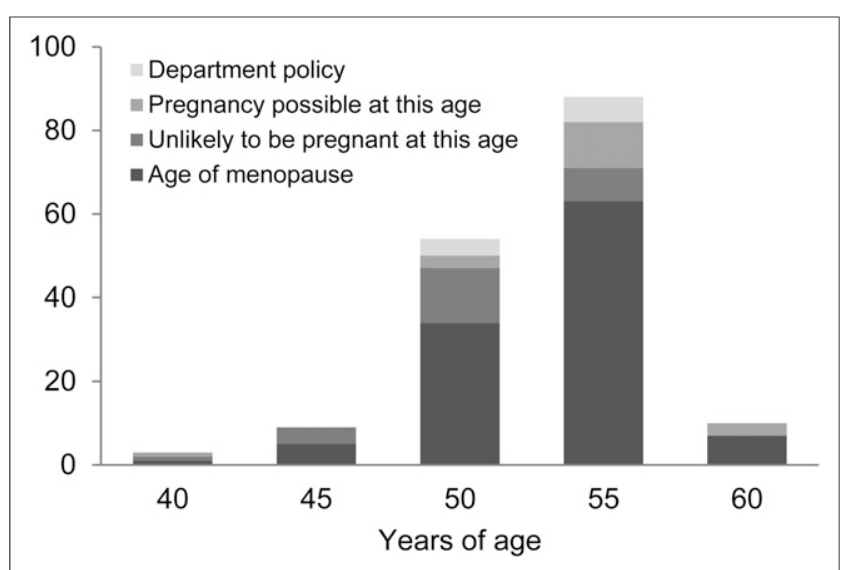

FIGURE 5. Rationale for maximum age.

The main reason cited for using pregnancy tests was if the patient was unsure or doubtful about her pregnancy status (45\%). Other reasons cited were for therapeutic-dose patients, if pregnancy could not be excluded, if the patient had had unprotected sex, if their LMP was late, if there had been more than $10 \mathrm{~d}$ since their LMP, if the patient thought they could be pregnant, and if the radiation dose from the procedure was considered high (Fig. 6).

\section{Radiation Risk to Fetus}

Participants were asked to rank 8 diagnostic nuclear medicine procedures as to their risk to a fetus from ionizing radiation, with a ranking of 1 being the most risk and a ranking of 8 being the least risk. Only 18 of $238(8 \%)$ participants correctly ranked all procedures according to fetal dose estimates reported by United Nations Scientific Committee on the Effects of Atomic Radiation (1). However, most participant responses were correctly ranked if the procedures were classified by relative risk as high (rank 1-3), 77\% correct; medium (rank 4, 5), 66\% correct; and low (rank 6-8), 75\% correct (Table 2).

Table 2 displays the participant rankings for each procedure (i.e., 171 participants indicated that ${ }^{18} \mathrm{~F}-\mathrm{FDG}$ PET/ CT was the highest-risk and 20 participants indicated that it was the lowest-risk procedure). The numbers marked by an asterisk show the correct ranking for each procedure which was also correctly identified by most participants.

TABLE 1

Routine Questions

\begin{tabular}{lcc}
\hline \multicolumn{1}{c}{ Question } & Verbal $(n=137)$ & Written $(n=69)$ \\
\hline LMP & $122(89)$ & $64(93)$ \\
Contraceptive use & $76(55)$ & $28(41)$ \\
$\begin{array}{l}\text { Menopause or } \\
\text { hysterectomy }\end{array}$ & $98(72)$ & $36(52)$ \\
\hline
\end{tabular}

Data in parentheses are percentages.

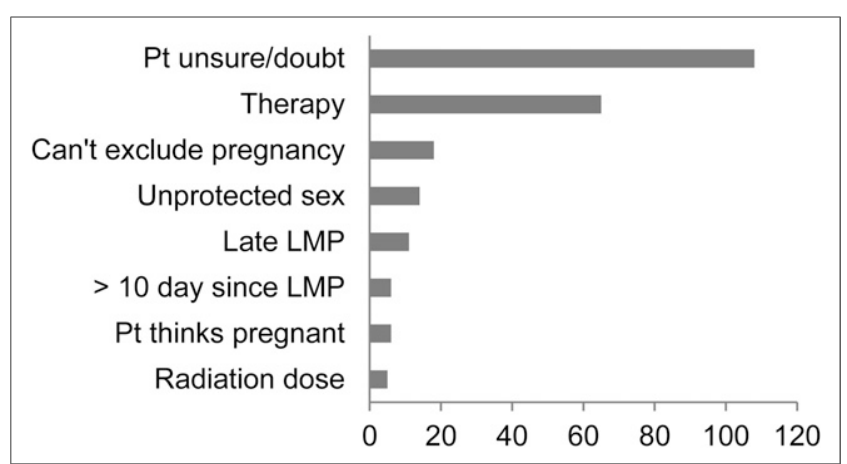

FIGURE 6. Reported circumstances for using pregnancy tests. Pt $=$ patient.

\section{DISCUSSION}

Ionizing radiation is potentially harmful to a developing fetus. Although diagnostic nuclear medicine procedures use relatively small amounts of radiation, it is still imperative to determine whether a patient might be pregnant before commencing the procedure. Radiation protection documents such as ICRP 84 (3) and ARPANSA 14.2 (4) provide recommendations regarding the protection of patients undergoing diagnostic nuclear medicine procedures. These documents recommend that all females of childbearing age be questioned regarding their pregnancy status before administration of any radiopharmaceuticals. They do not, however, give instructions on what constitutes the childbearing age range, how to question the patient, or when to use pregnancy testing.

\section{Policy and Regulations}

This study revealed a low awareness of government and international regulations concerning pregnancy and medical radiation. Most participants reported that they had not read the relevant sections regarding pregnancy and diagnostic procedures in ICRP 84 (3) or ARPANSA 14.2 (4), which correlates with the findings from a previous interview study conducted by the authors (7).

ICRP publication 84, Pregnancy and Medical Radiation, is one of the primary international documents detailing recommendations for the use of ionizing radiation in pregnant and potentially pregnant women. Chapter 6 discusses radiation protection applicable to nuclear medicine. It states that for "women of childbearing age, the possibility of pregnancy and the justification for the examination should be considered." It recommends that patients be "carefully interviewed" to determine their pregnancy status and that "discretion" is required in the case of adolescents. It also states that "advisory notices should be posted" in the nuclear medicine department.

ARPANSA 14.2, Safety Guide for Radiation Protection in Nuclear Medicine, was published by the Australian government to provide advice on radiation practice. Section 5 discusses the protection of the embryo or fetus. It states that "all female patients of child bearing age" should be ques- 
TABLE 2

Radiation Risk to Fetus

\begin{tabular}{|c|c|c|c|c|c|c|c|c|c|}
\hline \multirow[b]{2}{*}{ Relative risk } & \multirow[b]{2}{*}{ Procedure } & \multicolumn{8}{|c|}{ Ranking (highest risk $\rightarrow$ lowest risk) } \\
\hline & & 1 & 2 & 3 & 4 & 5 & 6 & 7 & 8 \\
\hline \multirow[t]{3}{*}{ High } & ${ }^{18} \mathrm{~F}-\mathrm{FDG} \mathrm{PET} / \mathrm{CT}$ & $171^{*}$ & 32 & 11 & 6 & 7 & 6 & 3 & 20 \\
\hline & Bone scanning with SPECT/CT of lumbar spine & 37 & $131^{*}$ & 35 & 18 & 7 & 9 & 14 & 2 \\
\hline & 99mTc-sestamibi myocardial perfusion scan & 18 & 49 & $102^{*}$ & 31 & 21 & 12 & 13 & 5 \\
\hline \multirow[t]{2}{*}{ Medium } & Gated heart pool scan & 2 & 9 & 34 & $85^{*}$ & 73 & 37 & 9 & 2 \\
\hline & Bone scan & 1 & 9 & 38 & 81 & $94^{*}$ & 26 & 4 & 1 \\
\hline \multirow[t]{3}{*}{ Low } & Renal perfusion scan & 5 & 7 & 23 & 21 & 30 & $105^{\star}$ & 47 & 14 \\
\hline & Ventilation and perfusion lung scan & 10 & 12 & 4 & 6 & 12 & 37 & $93^{*}$ & 81 \\
\hline & 99mTc thyroid scan & 14 & 9 & 7 & 5 & 4 & 19 & 68 & $127^{\star}$ \\
\hline
\end{tabular}

${ }^{*}$ Correct ranking for each procedure, which was also correctly identified by most participants.

tioned regarding their pregnancy status "immediately before the procedure"; the reason for asking should be explained to the patient "to avoid the patient taking offense and not answering fully"; this type of discussion "requires tact and discretion"; asking teenagers may be a "sensitive issue"; an interpreter may be required when language barriers exist; and that patient history alone "may not be reliable." The guide lists several circumstances for when the likelihood of pregnancy is low or physically impossible. These include hysterectomy, tubal ligation, normal menstrual period within the last $10 \mathrm{~d}$ (if the patient has regular menstrual periods), contraceptive measures having been taken (providing they have been taken regularly), and no sexual relationship for several months. If pregnancy status is uncertain, "the nuclear medicine specialist should be consulted" to decide whether to postpone the study, perform a pregnancy test, or continue with the study. This decision may be influenced by the "level of radiation dose" from the procedure.

\section{Current Practice}

The age range to include for questioning about pregnancy has not been clearly defined. The use of the term "childbearing age" in radiation protection documents refers to any female capable of reproduction. Both the age at menarche and the age at menopause are individual and can vary greatly among women.

The age at menarche is primarily influenced by genetic factors but also by nutrition, geography, and altitude (8). In a study investigating age at menarche in 34 countries, Currie et al. (9) reported that "in $95 \%$ of individuals, age at menarche ranged between 10 y 6 mo and 14 y 11 mo." Several studies $(8,10,11)$ discuss whether there has been a decline in the age of menarche over the past $50 \mathrm{y}$. Declining age at menarche has been associated with obesity (11). Some studies report a decline in age from 13 to $12 \mathrm{y}$ since the early 20th century, but that in the latter part of the century age of menarche appears stable $(8,10)$. Posner $(10)$ reports that the "reference range for onset of menarche in the Unites States is now considered 10-14 y."

The age at natural menopause varies greatly among women. Factors believed to influence age at menopause include ethnicity, location, smoking, body mass index, physical activity, and number of children (12). A large Australian study of 5,961 twin females by Do et al. reported the median age at natural menopause as $51 \mathrm{y}$ (13). Another study of 5,288 women by Dratva et al., conducted in 9 European countries, reported the median age at menopause as $54 \mathrm{y}$ (12). Surgically induced menopause (hysterectomy or tubal ligation) is associated with earlier timing of menopause (12).

Participants in our study reported using age at menarche as the rationale for applying a particular minimum age to question patients from $10 \mathrm{y}$ and up to $16 \mathrm{y}$ of age. Age of menopause was used as the rationale for choosing a particular maximum age between 40 and $60 \mathrm{y}$. These choices imply a lack of knowledge regarding these 2 aspects of female physiology and reproduction and possible reliance on personal interpretation and experience. Because both menarche and menopause can occur at different ages in individual women, it may be prudent to first ask whether the patient has either begun menstruating (for young teenagers) or completed menstruating (for women over 50 $y$ of age) or ask the date of the LMP, rather than beginning with a question asking if the patient might be pregnant.

Although $65 \%$ of participants indicated they had a written department policy for questioning patients, few stated this as their rationale for the minimum or maximum age to question patients. This may be due to departmental policies that use wording similar to that used in radiation protection documents, which refer to women of childbearing age but do not define the age range or how to determine who is classed as childbearing. For minimum age, many participants commented that selection of patients to question was dependent on the patient's perceived maturity, implying that some nuclear medicine per- 
sonnel make assumptions about the patient when determining pregnancy status.

The American College of Radiology recommends that "all patients of menstrual age (typically ages 12 through 50 y) should be questioned about pregnancy status using a standardized form or through direct questioning by the technologist." In addition, it recommends the use of a standardized form to ensure uniformity in the questioning process (5). Variations in practice can lead to individual patients being treated differently and consequently not receiving the same level of care. Gabel and Shipan state that reducing practice variation is "a fundamental issue for increasing the quality of health care" (14). The development of a standardized form to determine pregnancy status may assist nuclear medicine personnel in ensuring that the appropriate patients are asked appropriate questions. Our study showed a wide variation in the methods used to determine which patients to question and which questions they were asked. Verbal questioning of the patient is still predominately used, with or without the addition of the patient's signature, and this may be a contributing factor to inconsistencies in practice.

Pregnancy testing is not routinely used before diagnostic nuclear medicine imaging procedures. Pregnancy testing for all female patients would be unnecessary, timeconsuming, and expensive (7). Urine pregnancy tests are relatively inexpensive and easy to use; however, if used before the date of missed menses they have been reported to have a high false-negative rate (15). The most commonly reported circumstance for using pregnancy testing is when the patient is unsure of her pregnancy status. The reliability of patient history and self-assessment of pregnancy status has been reported with conflicting results $(16,17)$. Minnerop et al. (18) investigated the reliability of patient assessment to exclude pregnancy and found that women are able to exclude pregnancy. They reported a $100 \%$ negative predictive value for women stating pregnancy was impossible. They suggest that history of tubal ligation, intrauterine device, or oral contraceptive use should not be relied on to exclude pregnancy. Minnerop et al. recommended using patient history and suspicion of pregnancy and weighing these against the risk associated with an undiagnosed pregnancy to decide if a pregnancy test is warranted.

A small number of participants indicated that they used pregnancy testing if the radiation dose from a procedure was considered high. This is recommended in ARPANSA 14.2 (4); however, a definition of higher dose is not provided. Diagnostic nuclear medicine procedures are generally considered low-dose procedures, with most ${ }^{99 \mathrm{~m}} \mathrm{Tc}$ procedures giving an effective dose of less than $10 \mathrm{mSv}$ to an adult patient (1). Because the dose is quite low for these procedures, it may be sufficient to rely on patient selfassessment of pregnancy status and to use pregnancy testing only where there is concern or doubt surrounding the patient's responses. Other procedures that use longer-lived radioisotopes, positron emission, or hybrid CT imaging may provide a higher effective dose to the patient and therefore would warrant the use of pregnancy testing before administration of the radiopharmaceutical.

\section{Radiation Risk to Fetus}

Estimation of fetal dose from maternal examinations can be difficult. Calculation of fetal dose estimates must include factors such as the physical properties of the radionuclide, the administered activity, and the stage of fetal development. The chemical and biologic properties of the radiopharmaceutical must be considered to determine the amount of placental transfer and biodistribution in fetal tissues. External irradiation of the fetus from maternal organs also adds to the dose (19). The fetal doses from most diagnostic nuclear medicine procedures are "much lower than the levels where developmental and neurologic effects are known to occur" (4). However, the dose levels have been reported to be associated with "a slightly increased risk of childhood cancer or leukaemia" (4). Although the risks to the fetus are low, anxiety and distress may be caused if a woman is irradiated and subsequently finds out she is pregnant. The participants in this study ranked several commonly performed procedures according to their radiation risk to the fetus, and although only a small number of participants correctly ranked every procedure, most participants were able to identify the risks as low, medium, and high.

\section{CONCLUSION}

The results of the survey suggest that nuclear medicine personnel in Australia and New Zealand use a variety of methods to determine which female patients to question, and how they are questioned, regarding their pregnancy status before diagnostic imaging procedures. Although there is a lack of knowledge concerning radiation protection guidelines for the protection of the fetus, a good level of knowledge of the relative fetal risk from commonly performed procedures was demonstrated. We recommend that a standardized practice guideline be developed to ensure consistent practice and to reduce the possibility of any unnecessary fetal irradiation.

\section{DISCLOSURE}

No potential conflict of interest relevant to this article was reported.

\section{REFERENCES}

1. UNSCEAR. UNSCEAR 2008 Report. Vol. II. Sources and Effects of Ionizing Radiation. New York, NY: United Nations; 2008.

2. De Santis M, Cesari E, Nobili E, Straface G, Cavaliere AF, Caruso A. Radiation effects on development. Birth Defects Res C Embryo Today. 2007;81:177-182.

3. International Commission on Radiological Protection (ICRP). Pregnancy and medical radiation, ICRP publication 84. Ann ICRP. 2000;30.

4. Australian Radiation Protection and Nuclear Safety Agency (ARPANSA). Safety Guide for Radiation Protection in Nuclear Medicine. Vol. 14.2. Yallambie, Victoria, Australia: ARPANSA; 2008. 
5. American College of Radiology (ACR). ACR Practice Guideline for Imaging Pregnant or Potentially Pregnant Adolescents and Women with Ionizing Radiation. Reston, VA: ACR; 2008.

6. Schreiner-Karoussou A. A preliminary study of issues and practices concerning pregnancy and ionising radiation. Radiat Prot Dosimetry. 2009;135: 79-82.

7. James DJ, Cardew P, Warren-Forward H. Determining the pregnancy status of patients before diagnostic nuclear medicine procedures: the Australian experience. J Nucl Med Technol. 2011;39:220-225.

8. Allison CM, Hyde JS. Early menarche: confluence of biological and contextual factors. Sex Roles. 2013;68:55-64.

9. Currie C, Ahluwalia N, Godeau E, Nic Gabhainn S, Due P, Currie DB. Is obesity at individual and national level associated with lower age at menarche? Evidence from 34 countries in the health behaviour in school-aged children study. J Adolesc Health. 2012;50:621-626.

10. Posner RB. Early menarche: a review of research on trends in timing, racial differences, etiology and psychosocial consequences. Sex Roles. 2006;54: 315-322.

11. Walvoord EC. The timing of puberty: is it changing? Does it matter? J Adolesc Health. 2010;47:433-439.
12. Dratva J, Gomez Real F, Schindler C, et al. Is age at menopause increasing across Europe? Results on age at menopause and determinants from two populationbased studies. Menopause. 2009;16:385-394.

13. Do KA, Treloar SA, Pandeya N, et al. Predictive factors of age at menopause in a large Australian twin study. Hum Biol. 1998;70:1073-1091.

14. Gabel MJ, Shipan CR. A social choice approach to expert consensus panels. $J$ Health Econ. 2004;23:543-564.

15. Cole LA, Khanlian SA, Sutton JM, et al. Accuracy of home pregnancy tests at the time of missed menses. Am J Obstet Gynecol. 2004;190: $100-105$.

16. Ramoska EA, Sacchetti AD, Nepp M. Reliability of patient history in determining the possibility of pregnancy. Ann Emerg Med. 1989;18:48-50.

17. Strote J, Chen G. Patient self assessment of pregnancy status in the emergency department. Emerg Med J. 2006;23:554-557.

18. Minnerop MH, Garra G, Chohan J, Troxell R, Singer A. Patient history and physician suspicion accurately exclude pregnancy. Am J Emerg Med. 2011;29: 212-215.

19. National Council on Radiation Protection and Measurements (NCRP). NCRP Report No. 128: Radionuclide Exposure of the Embryo/Fetus. Bethesda, MD: NCRP; 1998. 